\title{
Prevalence of Candida spp. and age-related disparities amongst women presenting with vaginitis at the Obstetrics and Gynaecology (O\&G) Clinic in a Tertiary hospital in Port Harcourt, Nigeria
}

\author{
Chiaka Mbakwem-Aniebo, Anwuli Uche Osadebe, Eunice Athanasonny, Iheanyi Omezurike Okonko
}

\author{
Department of Microbiology, University of Port Harcourt, P.M.B. 5323, Choba, Nigeria.
}

\begin{abstract}
Background: Vaginitis, an infection of the lower genital tract in women, is known to be triggered by the overgrowth of the vagina's naturally occurring microorganisms.

Objective: This study looked at the prevalence of Candida spp. and age-related disparities amongst women presenting with vaginitis at the Obstetrics and Gynaecology (O\&G) clinic in a tertiary hospital in Port Harcourt, Nigeria.

Methods: One hundred high vaginal swabs were collected from pregnant and non-pregnant women and examined microscopically and microbiologically.

Results: Age-group 20-29 years had the highest incidence of candidal vaginitis. There was a higher occurrence of yeast cells in pregnant than in non-pregnant participants while the non-pregnant women had a greater level of bacterial cells. Forty (40) of the samples contained yeasts of Candida species representing a $40 \%$ prevalence. Three species of Candida were identified with C. albicans dominating. Of the 40 samples positive for Candida spp., 30 (75.0\%) were confirmed to be C. albicans. The remaining isolates were C. tropicalis (15.0\%) and C. parapsilosis (10.0\%).
\end{abstract}

Conclusion: The findings in this study would play a role in the future management of Candida-induced vaginitis.

Keywords: Candida, epidemiology, prevalence, vaginitis, vulvovaginal candidiasis.

DOI: https://dx.doi.org/10.4314/ahs.v20i1.9

Cite as: Mbakwem-Aniebo C, Osadebe AU, Athanasonny E, Okonko IO. Prevalence of Candida spp. and age-related disparities amongst women presenting with vaginitis at the Obstetrics and Gynaecology (O\&G) Clinic in a Tertiary hospital in Port Harcourt, Nigeria. Afri Health Sci. 2020;20(1):51-8. bttps://dx.doi.org/10.4314/abs.v20i1.9

\section{Introduction}

Vaginitis is an infection of the lower genitals in women often caused by yeasts and other fungi. It is known to be triggered by the overgrowth of Candida in the vagina and is a common infection of the female genital tract ${ }^{1}$. It may also be caused by Trichomonas vaginalis, Gardnerella vaginalis and Chlamydia trachomatis or a combination of these various microbial groups. When caused by Candi$d a$ spp., it is often referred to as vulvovaginal candidiasis (VVC) and is characterised by itching, curd-like vaginal discharge and erythema ${ }^{2,3}$. Studies show that almost $70 \%$ of females will suffer candidal vaginitis at least once in their lifetime while in approximately $50 \%$ of women, the

\section{Corresponding author: \\ Iheanyi O Okonko, \\ Department of Microbiology, \\ University of Port Harcourt, \\ P.M.B. 5323, Choba, Nigeria.}

E-mail address: iheanyi.okonko@uniport.edu.ng infection will occur multiple times. This figure goes up to $75 \%$ in women of child-bearing age $\mathrm{e}^{1,3,4}$. The factors that predispose women to VVC include hormonal fluctuation, such as occur during pregnancy and the menstrual cycle, diabetes, sustained antibiotic use and use of oral contraceptives $^{3,5}$. It has been reported, however, that 5\%-10\% of apparently healthy women experience persistent vaginal candidiasis devoid of defined predisposing factors ${ }^{6}$.

Candida spp. are part of the natural vaginal flora in $20.0 \%$ $-50.0 \%$ of healthy women but may become pathogenic under certain conditions including the presence of invading pathogens or biochemical changes in the vaginal environment making them common aetiologic agents of vaginitis ${ }^{1}$. Invading pathogens alter the normal microflora of the vagina while biochemical changes in the vagina stimulate the rapidproliferation of the natural Candida population, enhance their attachment to the epithelial cells of the vagina and promote germination of daughter yeast cells so that normal asymptomatic colonisation becomes symptomatic Candida infection ${ }^{1,7}$. The evidence 
further suggests that the protracted antimicrobial use causes irritation in body tissues making them susceptible to penetration and adherence by Candida spp. ${ }^{8}$.

Beyond the epidemiological import of documenting trends in pathogenic prevalence and noting the likely sources of infection, recording these changes has strong implications for treatment regimens and management. In Nigeria, there is a paucity of data on the distribution of candidal vaginitis and the species involved. There is only limited work on candidal vaginitis in South Southern Nigeria. This study sought to establish the different Candida species involved in vaginitis in women attending the Obstetrics and Gynaecology $(O \& G)$ clinic in a tertiary hospital in Port Harcourt, Nigeria.

\section{Materials and methods Study design}

This was a cross-sectional study employing both biological and demographic data. The study population consisted of adult females attending the Obstetrics \& Gynaecology clinic of the University of Port Harcourt Teaching Hospital (UPTH), Rivers State, Nigeria with symptoms of vaginitis.

The sample size of 100 was derived using the formula:

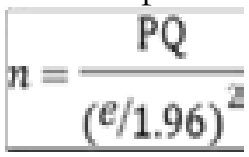

Where $\mathrm{n}=$ sample size; $\mathrm{P}^{*}=$ working proportion $=$ $6.89 \% ; \mathrm{Q}=100 \_\mathrm{P}=100 \_6.89=93.11 \%$; $\mathrm{e}=$ margin of sampling error tolerated (5\%), at $95 \%$ degree of confidence.

*The working proportion $(\mathrm{P})$ was determined by the prevalence of Candida-induced VVC based on other studies in the region.

\section{Ethical considerations}

All the participants provided informed consent for inclusion in the study and were assured that all the information provided would be used solely for the purposes of this study and treated confidentially. Ethical approval was given by the UPTH.

\section{Patient eligibility and inclusion criteria}

All participants had to have presented with symptoms of vaginitis. Women who voluntarily consented to participate were considered eligible and recruited for the study. All those who did not assent or did not have symptoms were not included.

\section{Sample collection}

A total of 100 high vaginal swabs (HVS) samples were collected from 60 symptomatic pregnant and 40 symptomatic non-pregnant females between the ages of $20-$ 49 years attending the $\mathrm{O} \& \mathrm{G}$ clinic of the UPTH. Samples were collected from patients with at least one of the following symptoms: vaginal discharge, itching and/or burning sensation. The data were analysed anonymously.

\section{Microscopy of HVS Smears}

Microscopic examination of the high vagina swab smears was via wet preparation. A drop of $10.0 \%$ potassium hydroxide solution was added to a smear of HVS on a clean glass slide, covered with a coverslip and then examined under the microscope. The wet preparation was done to check for motile organisms like Trichomonas vaginalis, yeast cells, pus cells, epithelial cells and bacterial cells.

\section{Isolation of bacteria}

The presence of bacteria in the swab samples was examined via isolation on Nutrient Agar and Mannitol Salt Agar. The swab samples were suspended in sterile normal saline and inoculated onto agar plates following serial dilution. Incubation was at $37^{\circ} \mathrm{C}$ for 24 hours. Biochemical testing was employed in the identification of the isolates. The tests carried out included sugar fermentation tests, oxidase test, $\mathrm{H}_{2} \mathrm{~S}$ production, citrate utilisation, motility, indole formation, urea hydrolysis, catalase test, coagulase test, lysine decarboxylase and lysine deaminase production, Methyl Red test, Voges Proskauer test and Gram's staining 9

\section{Characterisation of Candida species}

The swabs were immediately streaked on Sabouraud dextrose agar (SDA) supplemented with chloramphenicol $(500 \mathrm{mg} / \mathrm{ml})$ and incubated at $37^{\circ} \mathrm{C}$ for 48 hours, then examined for the cream coloured pasty colonies indicative of yeast colonies. Identification was done using direct microscopy, culture and biochemical methods and the prevalence determined. Cultures identified as yeast were tested for germ-tube and chlamydospore formation using human serum and corn meal agar respectively. 


\section{Germ tube test}

A small portion of the yeast was inoculated into human serum in a test tube and incubated at $37^{\circ} \mathrm{C}$ for 3 hours. A drop of the serum-yeast suspension was then examined under the microscope using lactophenol blue stain?

\section{Statistical analyses}

Statistical Package for the Social Sciences (SPSS) ${ }^{\circledR} 21.0$ (International Business Machines Corporation (IBM), NY, USA) was used to analyse the data. Statistical significance of data sets was determined at $\mathrm{p} \leq 0.05$.

\section{Results}

Fig. 1 depicts the age distribution in the study. The results of the microscopic examination of the samples are shown in Fig. 2. Age group $20-24$ showed the greatest numbers of yeast, epithelial and pus cells. Trichomonas vaginalis was only found in this group as well. Comparisons between the two groups showed that there was a higher occurrence of yeast cells in pregnant than in non-pregnant participants while the non-pregnant women had a greater number of bacterial cells (Fig. 3).

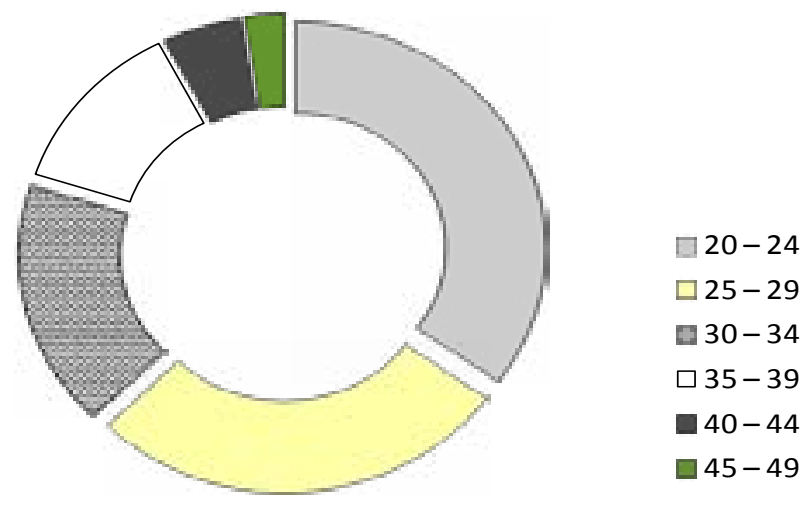

Figure 1: Age distribution of participants

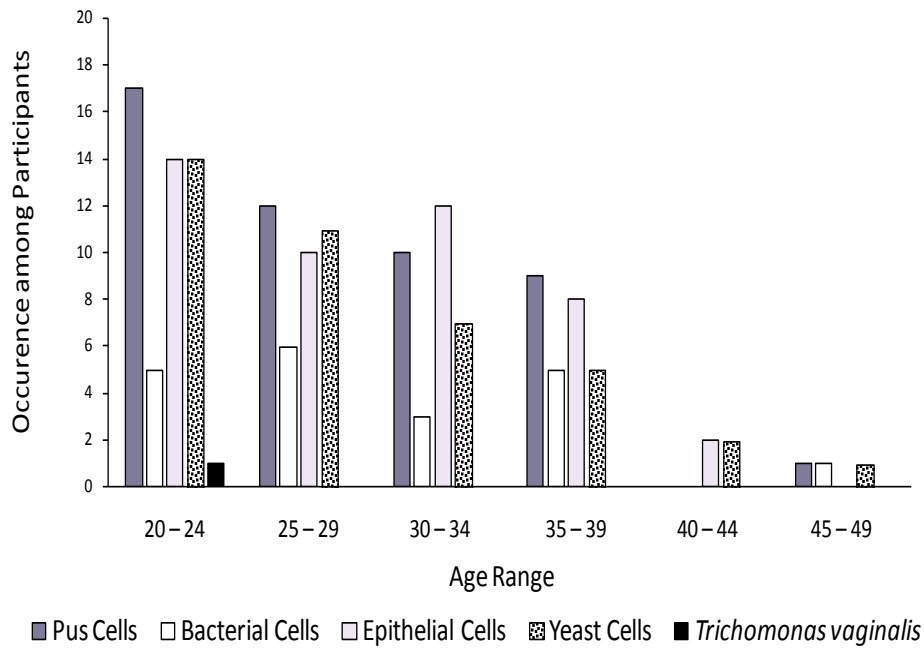

Figure 2: Microscopy of high vaginal swab samples 


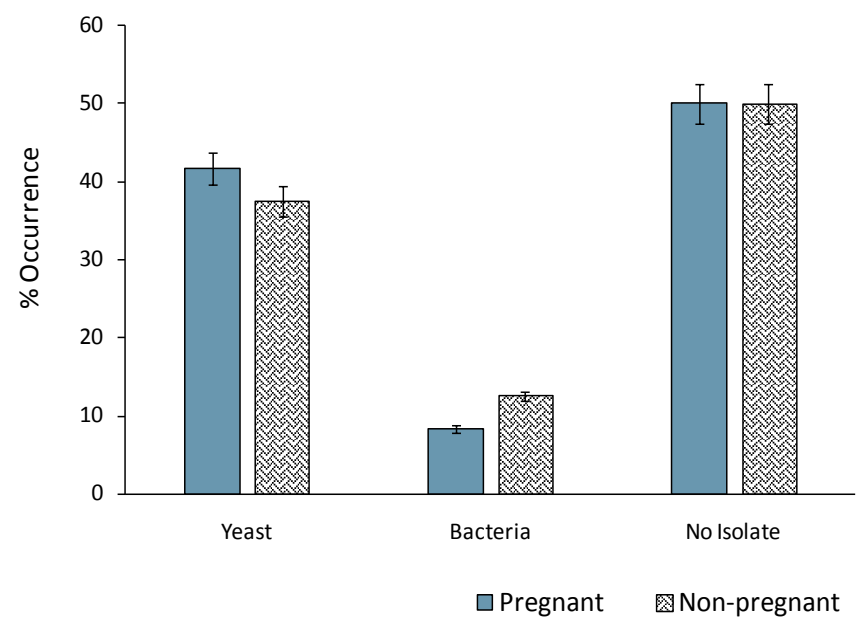

Figure 3: Observed microscopy amongst pregnant and non-pregnant participants

There was a $40.0 \%$ prevalence of Candida-induced vaginitis in this study with Candida albicans being the dominant aetiologic agent. The prevalence of non-C. albicans species was $10.0 \%$. Of the one hundred samples collected, forty (40) showed growth of Candida spp., forty (40) showed no microbial growth while twenty (20) showed bacterial growth. Following the germ tube and sugar fermentation tests, it was observed that $30(75.0 \%)$ of the 40 isolates were Candida albicans 6(15.0\%) were Candida tropicalis and $4(10.0 \%)$ were Candida parapsilopsis. The observed isolates are presented in Fig. 4 while the occurrence of the Candida isolates is illustrated in Fig. 5 among some bacterial isolates encountered which included Staphylococcus aureus and Streptococcus sp. (Fig. 4). The results also showed that individuals aged 20-29 years had the greatest number of Candida isolates but ages 40-49 had the highest frequency of isolation (Figs. 6 and 7).

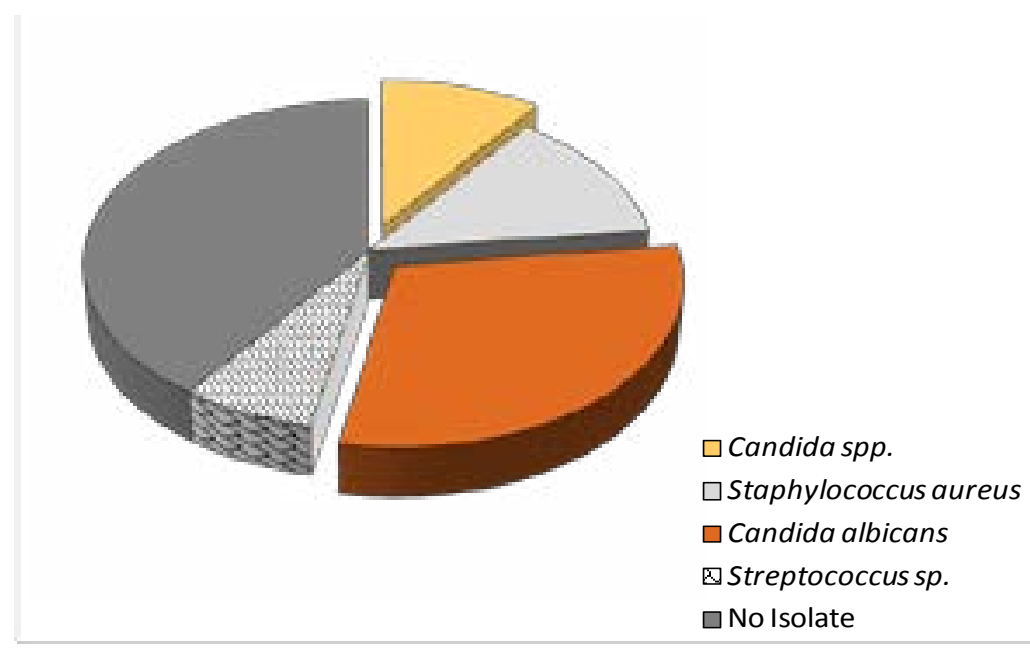

Figure 4: Distribution of observed isolates 


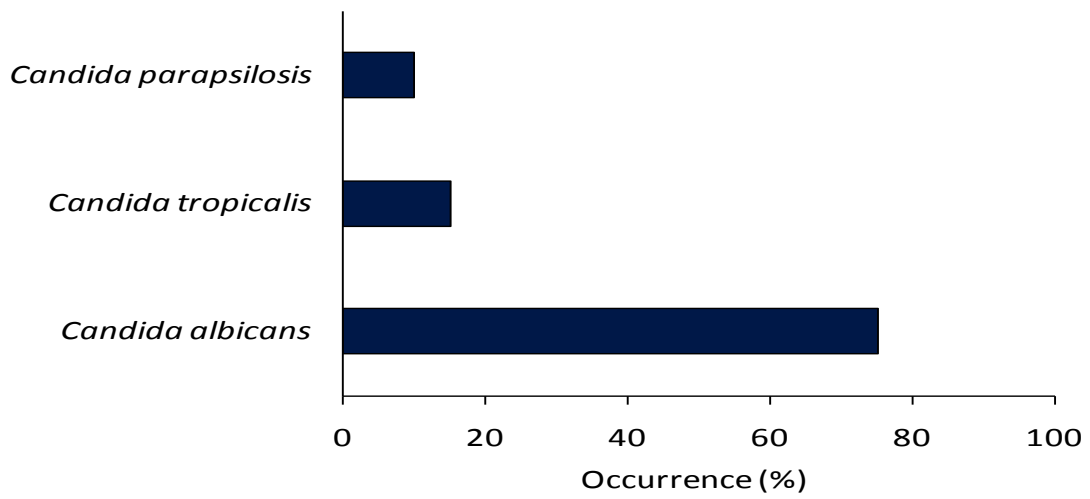

Figure 5: Occurrence of candida species isolated

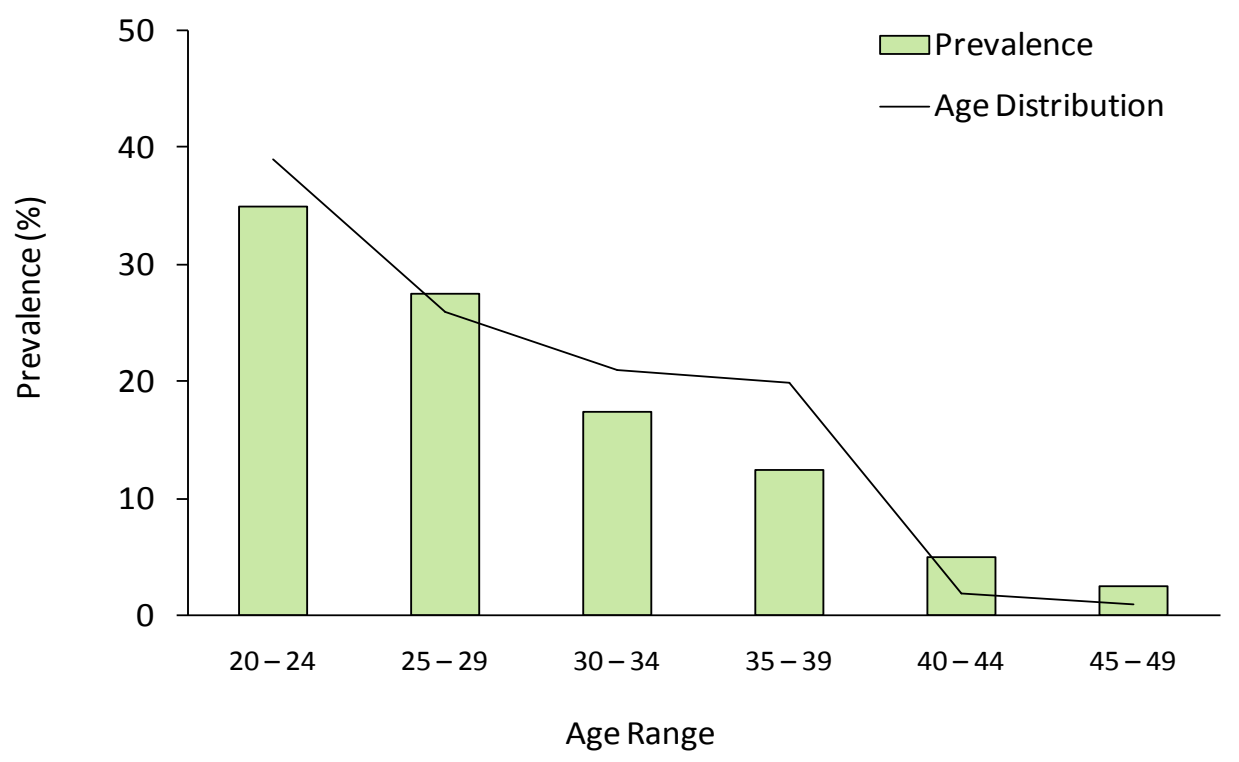

Figure 6: Prevalence of candida-induced vaginitis according to age showing age distribution 


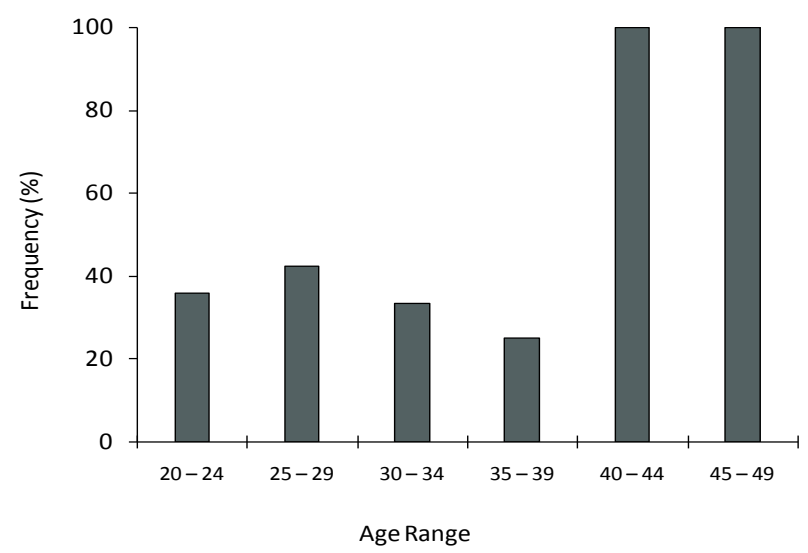

Figure 7: Frequency of isolation of candida spp. with respect to age

\section{Discussion}

The results showed that women in age group 20-29 years had more Candida-induced vaginitis and were also the dominant group in the clinic since they had the greatest number of positive Candida isolates. This study further showed that the age group $20-29$ years had the greatest prevalence of Candida spp. Some studies have shown that the prevalence of Candida increased significantly in pregnant women below 35 years but decreased in pregnant women above 35 years $^{3,7}$. Another similar study also reported the prevalence of C. albicans and non-C. albicans to be $6.5 \%$ and $7.5 \%$ respectively out of 200 individuals predominantly aged 20 to 30 years ${ }^{1}$. Findings from Alo et al. $^{10}$ were different as they observed a higher prevalence in the 36 to 40 age-group while the age-group 20 -25 years had the lowest prevalence. The differences in the prevalence of candidal vaginitis have been linked to high sexual activity, use of contraceptives, poor hygiene and antibiotics/ drug abuse as is common in the younger, more sexually active age-groups ${ }^{11,12}$.

There are a wide variety of reports on the prevalence of candidal vaginitis. The $40.0 \%$ prevalence in this study is much lower than that recorded by a study in Kenya that obtained a $90.38 \%$ prevalence of candidal vaginitis in pregnant women attending antenatal clinic. In some studies, the prevalence of Candida albicans-induced vaginitis was placed at $70.0-80.0 \%{ }^{13,14}$. Other studies, however, recorded lower prevalences of $14.0-21.0 \%$ in both pregnant and non-pregnant women ${ }^{1,15-17}$. A study in Nnewi, Nigeria, obtained a comparable prevalence of $30.0 \%$, similar to the present study ${ }^{18}$ and in Ethiopia, a prevalence of $41.1 \%$ among 210 women $^{12}$. Several factors could be responsible for the wide disparity in prevalence figures. The environment is one such factor and it has been found to play a role in the occurrence and dissemination of Candida $s p p$. within hospital environments. One study found that the most commonly isolated non-C. albicans species in the hospital environment, including the coats of staff, were C. glabrata $(37.62 \%), C$. parapsilosis $(25.74 \%)$ and $C$. tropicalis $(16.86 \%)^{19}$. This agrees with the higher occurrence of C. parapsilosis over C. tropicalis in this study. The findings in this study suggest that Candida spp. were the most significant causative organism of vaginitis while other possible causative species constituted less than $20.0 \%$ of the condition. Trichomonas vaginalis was isolated from only one (1) swab sample. This result agrees with the work of Richter et al. $^{4}$ who found other causative agents to be less than $1.69 \%$ of isolates and Trichomonas vaginalis occurred in only one (1) out of 593 isolates. Pregnant women showed a higher incidence of Candida infection. The marginal difference between the numbers of yeast cells observed in pregnant women compared to the number in non-pregnant women is somewhat unexpected as pregnant women are generally known to be more prone to yeast infections mainly because of their immunocompromised state and the changes in the $\mathrm{pH}$ of their vaginas.

The dominance of Candida albicans is well established. The occurrence of Candida albicans in $71.0 \%$ of VVC cases has been reported ${ }^{4}$. In India, $C$. albicans dominated with a recorded prevalence as high as $80.0 \%-85.0 \%$ while in Ethiopia, C. albicans had a prevalence of $58.6 \%$ over other Candida species $(41.4 \%)^{12,20}$. Candida albicans, Candida tropicalis and Candida parasilopsis are commonly associated 
with VVC with $C$. parapsilosis having a higher prevalence than C. tropicalis as was found in the current study ${ }^{21-23}$; other studies also identified C. krusei, C. lusitaniae and $C$. glabrata in addition to the observed species ${ }^{3,4,12}$. C. parapsilosis has been highlighted as a predominant cause of vulvovaginal candidiasis behind $C$. albicans $^{24}$. In contrast to the findings of this study, Grillot ${ }^{25}$ opined that non- $C$. albicans VVC infections are most often due to Candida glabrata in $5.0-10.0 \%$ of cases and Candida tropicalis in less than $5 \%$ of cases. Dan et al. ${ }^{9}$ found that non- $C$. albicans species accounted for $44.5 \%$ in asymptomatic women, $19.4 \%$ in women with recurring vaginitis and $21 \%$ in women with chronic symptoms. These figures are similar to the figures obtained in this study where non- $C$. albicans species made up $25.0 \%$ of isolates and $10.0 \%$ of all individuals tested. Mishra et al. ${ }^{26}$ also validated $C$. albicans as the most common agent of UTIs and reproductory tract infection. Similar trends are recorded in the epidemiology of candidal vaginitis in studies in southern Nigeria ${ }^{11,27}$. The current findings, however, contradict an earlier report from Benin City, Nigeria that C. glabrata was the most commonly encountered Candida species in symptomatic VVC pregnant women ${ }^{28}$.

A rise in the implication of non-C. albicans groups, especially $C$. glabrata, in vaginitis has been noted ${ }^{21,29-32}$. This shift is probably due to the increased misuse of over the counter drugs and antibiotics. Non- $C$. albicans infection is reported to be commonly linked to chronic but asymptomatic or mildly symptomatic vaginitis ${ }^{21,30,33}$. Candida albicans-linked infection, on the other hand, tends to be more symptomatic ${ }^{9}$. This might explain the low numbers of non-C. albicans isolates found in this study since the investigation was carried out on women with symptomatic vaginitis.

\section{Conclusion}

Vulvovaginitis is considered a complex infection as it involves many naturally occurring microorganisms in the vagina. In this study, there was a higher occurrence of yeast cells in pregnant than in non-pregnant participants while the non-pregnant women had a greater level of bacterial cells. Forty (40) of the samples contained yeasts of Candida species representing a $40 \%$ prevalence of vulvovaginal candidiasis. The highest prevalence was found amongst women in the age group 20 - 29 years. Candida albicans was the most prevalent causative agent. Other non-C. albicans species implicated were C. parapsilosis and C. tropicalis. These findings should help in the effective management of Candida-induced vaginitis. Continued surveillance would greatly support the management of this largely opportunistic infection. Further research aimed at determining possible risk factors, preventive therapy and treatment regimens is encouraged.

\section{Conflict of interest}

None declared.

\section{References}

1. Emeribe A, Nasir IA, Onyia J, Ifunnanya AL (2015) Prevalence of vulvovaginal candidiasis among non-pregnant women attending a tertiary health care facility in Abuja Nigeria. Research and Reports in Tropical Medicine 6: $37-42$.

2. Rathod, S. D., Klausner, J. D., Krupp, K., Reingold, A. L. and Madhivanan, P. (2012) Epidemiologic Features of Vulvovaginal Candidiasis among Reproductive-Age Women in India. Infectious Diseases in Obstetrics and Gynecology, 2012: Article ID 859071.

3. Nelson M, Wanjiru, W, Margaret MW (2013) Prevalence of vaginal candidiasis and determination of the occurrence of Candida species in pregnant women attending the antenatal clinic of Thika District Hospital, Kenya. Open J. Med Microbiol. 3: $264-272$.

4. Richter S. S., Galask, R. P., Messer, S. A. and Hollis, R. J. (2005) Antifungal Susceptibilities of Candida Species Causing Vulvovaginitis and Epidemiology of Recurrent Cases. Journal of Clinical Microbiology, 43(5): 2155 - 2162.

5. Jawetz, E., Melrick, J. L., Adelbergs, B. A. (2007) Medical Microbiology. $24^{\text {th }}$ Ed. McGraw Hill, New York, USA.

6. Mitchell, H. (2004) Vaginal Discharge-Causes, Diagnosis and Treatment. Biomedical Journal, 328: 1306 - 1308. 7. Wright, M. (2014) Vaginal and vulval candidiasis online. Available at http://www.patient.co.uk/doctor/ vaginal-and-vulval-candidiasis, accessed $10^{\text {th }}$ June 2018.

8. Mbakwem-Aniebo, C. (2010) Medical Mycology - Yeasts of Medical Importance. Pearl Publishers, Port Harcourt, Nigeria.

9. Dan, M., Poch, F. and Levin, D. (2002) High rate of vaginal infections caused by non-C. albicans Candida species among asymptomatic women. Medical Mycology 40, 383-386.

10. Alo, M. N., Anyim, C., Onyebuchi, A. K., Okonkwo, E.C. (2012) Prevalence of asymptomatic co-infection of candidiasis and vaginal trichomoniasis among pregnant 
women in Abakiliki, South Eastern Nigeria. J. Nat. Sci. Res. 2(7): $87-91$.

11. Akortha, E, Chikwe O, and Nwaugo, O. (2009) Antifungal Resistance among Candida Species from Patients with Genitourinary Tract Infection Isolated in Benin City, Edo estate, Nigeria. African Journal of Microbiology Research, 3 (11): 694-699.

12. Bitew, A. and Abebaw, Y. (2018) Vulvovaginal candidiasis: species distribution of Candida and their antifungal susceptibility pattern. BMC Women's Health 18: 94.

13. Beck-Sague, C and Jarvis, W R (1993) Secular trends in the epidemiology of nosocomial fungal infections in the United States 1980-1990 National Nosocomial Infections Surveillance System. J Infect Dis 167, 1247 - 1251. 14. Fidel, P. L. Jr, Vazquez, J A, and Sobel, J D (1999) Candida glabrata: review of epidemiology, pathogenesis, and clinical disease with comparison to C. albicans. Clin Microbiol Rev 12, 80-96.

15. Mahandani JW, Ekate RR, Shrikhande AV. (1998) Diagnosis of discharge per vaginum. Indian J. Pathol. Microbiol., 41(4): 403 - 411.

16. Nandan D, Gupta, YP, Krishnan V, Harma A, Misra SK. (2011) Reproductive tract infection in women of reproductive age group in Sitapur/ Shahjahan-pur district of Uttar Pradesh. Indian J. Public Health 45(1): 8 - 13.

17. Aring BJ, Mankodi, PJ, Jasani JH. (2012) Incidence of vaginal candidiasis in leucorrhoea in women attending the OPD of gynaecology and obstetrics department. Int J Biomed Adv:; 3(12): 867 - 869 .

18. Okonkwo, N. J. and Umeanaeto, P. U. (2010) Prevalence of candidal vaginitis among pregnant women in Nnewi town of Anambra state, Nigeria. Afr. Res. Rev. 4(4): $539-548$.

19. Savastano, C., de Oliveira Silva, E., Gonc'alves, L. L., Nery, J. M., Silva, N. C., Tranches Dias, A. L. (2016) Candida glabrata among Candida spp. from environmental health practitioners of a Brazilian Hospital. Brazilian Journal of Microbiology 47: 367 - 372.

20. Singh, S., Sobel, J. D., Bhargava, P., Bolkov, D. and Vazquez, J. A. (2002) Vaginitis Due to Candida krusei: Epidemiology, Clinical Aspects, and Therapy. Clinical Infectious Diseases 35: 1066 - 1070

21. Spinillo A, Capuzzo E, Gulminetti R, Marone P, Colonna L, Piazzi G. (1997) Prevalence and risk factors for fungal vaginitis caused by non-albicans species. $A m \mathrm{~J} O b$ stet Gynecol; 176: 138 - 141.

22 Trama, J P, Adelson, M E, Raphaelli, I, Stemmer, S M, and Mordechai, E. (2005) Detection of Candida species in vaginal samples in a clinical laboratory setting. Infect $D$ is Obstet Gynecol 13, 63 - 67.

23 Nyirjesy, P, Alexander, A B, and Weitz, M V (2005) Vaginal Candida parapsilosis: pathogen or bystander? Infect Dis Obstet Gynecol 13, 37-41.

24 van Asbeck, E. C., Clemons, K. V. and Stevens, D. A. (2009) Candida parapsilosis: a review of its epidemiology, pathogenesis, clinical aspects, typing and antimicrobial susceptibility. Critical Reviews in Microbiology, 35(4): 283 309.

25 Grillot R. (1996) Mycosis genitals. Les mycoses humaines: demarche diagnostique. Paris: Elsevier, pp116 124.

26 Mishra M, Agrawal, S, Raut S, Kurhade AM, Powar RM (2014) Profile of yeasts isolated from urinary tract of catheterised patients. J. of Clin. Diagn Res. 8: $44-46$.

27 Sehgal, S. (1990) Epidemiology of male Urithritis in Nigeria. Journal of Tropical Medicine Hygiene, 93: 151 - 152. 28 Okungbowa, F., Isuehuemhen, O. and Dede, A. (2003) The Distribution, Frequency of Candida Species in the Genitourinary Tract among Symptomatic individuals in Nigeria cities, Revised Iberoam Microbiology, 20(2): 60 $-63$

29 Horowitz B. J., Giaquanta D. and Ito S. (1992) Evolving pathogens in vulvovaginal candidiasis: implications for patients cure. J Clin Pharmacol., 32: 248-255.

30 Nyirjesy, P., Seeney, S. M., Grody, M. H. T., Jordan, C. A. and Buckley, H. R. (1995). Chronic fungal vaginitis: the value of cultures. Am. J. Obstet. Gynecol. 173:820 - 823. 31 Sobel, J. D. and Chaim, W. (1997) Treatment of Torulopsis glabrata vaginitis: retrospective review of boric acid therapy. Clin. Infect. Dis. 24: 649 - 652.

32 San Miguel, L G, Cobo, J, Otheo, E, Sanchez-Sousa, A, Abraira, V, and Moreno, S. (2005) Secular trends of candidemia in a large tertiary-care hospital from 1988 to 2000: emergence of Candida parapsilosis. Infect Control Hosp Epidemiol 26: 548 - 552.

33 Spinillo A, Capuzzo E, Nicola S, Baltaro F, Ferrari A, Monaco A. (1995) The impact of oral contraception on vulvovaginal candidiasis. Contraception 51(5): 293 - 297. 